Repères $\mathrm{n}^{\circ} 7$, Langage et images, INRP, 1993

ISBN 2-7342-0493-2

\title{
INFLUENCE CE L'AUdIOVISUEL SUR LES PRODUCTIONS ORALES ET ÉCRITES D'ÉLÈVES DE CM2
}

Marie-Christine POUDER

CNRS-URA1031 - Paris V

41

\section{Résumé :}

A partir de l'observation de deux classes de primaire (CM1 et CM2) en atelier informatique et de l'étude des enregistrements de groupes d"enfants en situation d'écriture et de graphisme, nous montrons que l'univers sémantique à partir duquel les élèves créent leurs textes est fortement lié au monde de l'audiovisuel : jeux vidéo, séries, émissions de variétés, longs et courts métrages. De manière plus ponctuelle, leurs dialogues peuvent s'appuyer sur des genres télévisuels ou musicaux (interviews, chansons) ou rejoindre également des thèmes anciens de la tradition orale. Nous nous situons dans une perspective d'interactions et d'étayage entre les différentes sémiotiques de l'image, de l'oral et de l'écrit.

Nous nous inscrivons dans la thématique du numéro en nous positionnant dans une double perspective, acquisitionnelle et interactionnelle, par rapport à un domaine d'application particulier, l'utilisation du traitement de texte et de logiciels graphiques par des élèves d'école primaire (CM1et CM2).

\section{ACQUISITION EN INTERACTION, INTERACTION ENTRE SÉMIOTIQUES}

Parler de la relation oral / écrit / image à l'école élémentaire en psycholinguiste interactionniste et non en pédagogue ou didacticien nécessite de positionner ces termes les uns par rapport aux autres en prenant comme organisateur de base non pas ces éléments en eux-mêmes, en tant par exemple qu'ils s'opposeraient dans une analyse structurale en sémiologie, mais le sujet pour lequel ces termes organisent ou médiatisent une représentation.

1.1 Interaction adulte / enfant

Nous savons maintenant que le bébé entre très tôt dans le circuit de l'interaction verbale ; la coordination du regard entre lui-même et l'adulte organise déjà l'espace en du thématisé et de l'indistinct et le commentaire mimique et verbal de l'aîné fonctionne comme prédication à propos de ce qui est focalisé dans l'expérience (J.Bruner). 
A partir des fonctions matures à la naissance, le nourrisson va peu à peu voir ses facultés de représentation s'organiser en relation avec le développement

42

de ses capacités perceptives et grâce aux étayages !angagiers' et culturels qui vont humaniser ses perceptions proprio et extéroceptives. L'adulte va assumer sa position d'étayage en assurant ta nomination du monde, des états et des affects de l'enfant, i! est son porte-parole ; il le modèle par son organisation du temps et de l'espace, son rythme de vie et ses représentations, les routines langagières dans lesquelles il l'engage.

Comme l'avait déjà signalé Bakhtine et comme le précisent certains développements actuels de la pragmatique (F. François), nous apprenons tous à parler au sein de genres discursifs particuliers en entrant dans des rôles parallèles ou complémentaires et en intériorisant ces rôles (en répétant, répondant, questionnant, nous opposant,refusant,critiquant ...) et non en appliquant simplement des étiquettes verbales aux objets du monde. Des expériences récentes en neurolinguistique montrent d'ailleurs combien les termes acquis sont reliés associativement à leur contexte d'acquisition.

1.2, Le livre, un des objets du monde, medium de l'image et de l'écrit

Parmi ces objets justement, Certains vont prendre selon les pays et les coutumes une importance fondamentale. J.P. Sartre dans " Les mots " mentionne la fascination qu'exerçaient sur fui ces "pierres levées" sur ta table de nuit et dans la bibliothèque de son grand-père ... les livres. Dans la culture occidentale l'enfant est très tôt, c'est-à-dire dès la fin de sa première année de vie, en relation avec un livre de carton ou de tissu, des images en plastique ; cette relation est toujours médiatisée par l'adulte qui dans le meilleur des cas l'incite à porter son attention sur les objets représentés, à en saisir les caractéristiques physiques, les fonctions, à les faire passer d'un monde de représentation à l'autre (fiction/réalité, présent/absent, proche/lointain). Selon les habitudes interactives françaises, les mères initient très tôt les enfants au fonctionnement du récit à partir de la lecture des images puis des textes d'histoires (récit-annonce, reconnaissance et pérennité de l'actant principal, attente et plaisir de la continuité, finitude de la séquence, etc.).

II nous semble qu'à côté des modèles laboviens du récit comme caractéristiques de l'oral, nous pouvons reconnâ̂tre l'influence de cette interaction jeune enfant / livre / adulte dans les conduites langagières et linguistiques ultérieures de l'enfant. Le moment où ce dernier passe de la grande variété des possibilités de thématisations, descriptions et associations à partir de l'image, relativement insaturables, à la reconnaissance régulière des actants et des actions semble important pour le passage de l'oral à l'écrit. II est frappant de voir combien sont proches par exemple les procédés de définition des mères aux enfants de 2-3 ans et les types de définition donnés plus tard par les enfants de 4-5 ans. Les explications relèvent d'une plus grande idiosyncrasie de l'enfant par rapport à l'adulte.

Ces routines apparaissent dans un cadre socio-culturel donné ; les enquêtes faites en France auprès de mères africaines analphabètes montrent qu'elles ne prêtent pas à l'enfant des aptitudes aussi précoces que les mères 
françaises, et qu'eUes organisent avec leurs enfants autour de l'objet livre des scripts qui n'ont pas la même fonction que ceux des mères francophones alphabétisées. Ici aussi interviennent les représentations des adultes : représentations de ce qui est bon pour le développement de l'enfant, représentations des capacités de l'enfant, représentations de la relation au livre. Les mères françaises ont par rapport au livre des conduites pédagogiques au sens de conduites scolaires (dénommer, répéter, répondre, pointer, faire attention, mémoriser, etc. ).

Nous pouvons dire que l'objet livre, et à travers lui une certaine sémiotique de l'image fixe, du graphisme, du texte et de l'écrit au sens strict, apparaissent très précocement dans le monde de l'enfant, souvent même avant l'âge de l'école \{entre 2 et 3 ans). Le texte fait signe avant de faire lettre et l'écrit s'apprend par le biais de l'ora! et de l'image.

Les variantes intonationnelles, syntaxiques et lexicales qui marquent le discours de l'adulte lecteur orientent l'enfant vers une conscience de la spécificité de ce mode de codage de la langue. Nous voyons ici, selon les vues de l'école de prague comment la structuration de la langue orale peut en être consolidée (forme des pronoms, négation, concordance des temps, monologue ...) (note 1$)$.

\subsection{Du livre ou livre cassette à la vidéocassette}

L'autonomisation progressive des jeunes par rapport aux nouvelles technologies accentue et diversifie ce phénomène ; il est d'observation courante dans des milieux permissifs que les enfants utilisent très tôt un magnétophone, et même leur propre magnétophone pour lire des livres cassettes (dès 3 ans) et qu'ils usent assez rapidlement du magnétoscope familial pour regarder leurs cassettes vidéo. Si ces habitudes introduisent les enfants aux procédures d'accès aux nouvelles technologies, elles sont incomplètes sans un étayage adulte (explications, commentaires, focalisation, triage d'informations, finalisation, évaluation, indications éthiques ...) ; néanmoins elles font jouer les possibilités d'auto-renforcement et d'auto-apprentissage du code écrit.

\section{UN DOMAINE D'ACTION EN PRIMAIRE}

\subsection{Situation}

L'objet langue écrite et l'objet image se rencontrent dans notre champ, rattachés à l'utilisation de l'ordinateur. Nous avons ainsi eu l'occasion d'observer deux classes du primaire, une classe de CM1 et 1/2 classe de CM2 dans deux ateliers informatiques de la ville de Paris (note 2).

Les enfants du CM1 recevaient une initiation au Logo, à un traitement de texte (Le Rédacteur sur Atari), et à une série de jeux vidéo. Ceux de CM2 devaient écrire un texte long directement sur traitement de texte (Write sur compatible IBM), l'llustrer et construire une page de couverture avec un logiciel graphique. Tous les textes devaient mettre en scène un ordinateur en plus d'animés humains. 
Cette situation n'est pas encore très llabitueUe en France à ce nrveaude scolarisation malgré tes instructions offici'etfes prônant t'initiation à f'informatique et aux tschnotogi9s nouveUes. Dans tes éco!es, !esnanoréseaux sont désertés et !e'ur uti!isation dépend souvent de ta passion d'une directrice ou de !adéterm. . natk>n d'un instituteur et de quelques-uns de ses cortègues.

\subsection{Représentations}

Les enfants sont souvent plus à l'aise que les adultes dans leurs rapports aux outils informatiques, et leurs représentations de ces objets les replacent dans un univers familier et banalisé.

Interrogés par leurs enseignante ("à quoi te fait penser le mot ordinateur 7"), les élèves de CM1, même s'ils n'ont que peu d'expérience directe de l'outil, associent en fonction de ce qui s'en rapproche le plus pour eux, les jeux vidéo (8), la télévision ou le magnétoscope (7) ; viennent ensuite des évocations de machines électroniques (5), à calculer (5), à travailler (5), à écrire (5), à dessiner (1). L'ordinateur est bien ici démythifié, vécu comme une machine au service de l'homme, construite pour l'assister et le faire progresser ; une seule association sur 53 donne à l'ordinateur une figure humaine (note 3 ).

Les élèves de CM2 situent leur héros et son ordinateur dans un univers familier : maison des parents, appartement, chambre ... L'ordinateur est reçu en cadeau à l'occasion des fêtes de Noël ou d'un anniversaire. Quatre-vingt pour cent des parents de ces élèves possèdent chez eux un, sinon deux ordinateurs.

Ces jeunes sont à un âge où ils s'orientent avec enthousiasme et confiance vers les machines. E. Lage (note 4), dans sa 'recherche sur les représentations de l'utilisation de l'ordinateur chez les jeunes du primaire et du secondaire montre que l'élève du primaire passionné d'informatique, fille ou garçon, est perçu très positivement par ses congénères ; cette image se modifie avec l'âge et les adolescents ne considèrent déjà plus positivement qu'une jeune fille de 14-15 ans s'intéresse à l'ordinateur et à l'informatique.

Nous pouvons penser que l'investissement pour cette discipline sera également dépendant des représentations que s'en font les adultes, instituteurs et surtout Institutrices. 1

\subsection{Télévision et publicité}

Pour les enfants, le déjà connu, à partir duquel ils abordent l'écran et le clavier de l'ordinateur correspond à la télévision et au magnétoscope ; depuis leur petite enfance, ils ont passé souvent plusieurs heures par jour devant le poste familial, intégrant spots publicitaires, slogans, chansons, séries animées ou filmées. Dans un travail de recherche portant sur l'évaluation de spots publicitaires par des enfants (maternelle, CE1, CM2), Th. Savoyen montre que, déjà bien présente en maternelle, la culture télévisuelle de l'enfant se développe et $\mathbf{s}$ 'améliore au $\mathrm{CE} 1$ puis auCM2 : "Au niveau des lexèmes purement spécifiques aux spots publicitaires, les CM2 sont les seuls à évoquer les paroles, la chanson, le clip, le 
film, le scénario, les trucages ... le vocabulaire est plus étendu que pour les autres classes et les enfants y ont recours à bon escient, les enfants sont sensibles au comique, aux différentes perceptions gustatives, visuelles, auditives ; ils critiquent les mauvais doublages ou les actions incongrues (note $5)$. Ils comprennent la fonction de la publicité, ils savent qu'elle est conçue pour faire connaître des produits et donner envie de les acheter.

$\mathrm{Ce}$ bref exempte illustre que les enfants ne se contentent pas d'être passifs devant l'écran de télévision mais qu'ils apprennent à lire l'image et à s'interroger sur les relations référent-image-évaluation / présentation des produits.

\section{TEXTES ET SÉMIOTIQUES EN JEU}

Nous allons essayer de montrer maintenant combien les productions orales et écrites des enfants de CM2 sont, d'après nous, inspirées par l'audiovisuel télévisuel, filmique et musical ; néanmoins nous voudrions attirer l'attention auparavant sur le fait que, si sur le plan de la perception auditive ou visuelle, nous sommes bien, à la radio, à la télévision, au cinéma, dans les domaines de l'oral ou de l'image, il n'en est pas tout à fait de même sur le plan de la facture et de la genèse du produit. Dans ce domaine également nous pensons qu'il y a une interaction entre les sémiotiques.

Nous vivons dans un oral scripturalisé, dans une écriture oralisée et nos images, si elles s'offrent à nous ou se dérobent en fonction de notre perception, sont parfois aussi construites selon une rationalité de l'écrit. Le scénario d'un film est le plus souvent écrit et mémorisé par des acteurs, même si une part non négligeable est laissée par certains metteurs en scène à l'imagination et à l'improvisation. Les présentateurs des journaux télévisés préparent soigneusement leurs prestations orales à partir de journaux, de reportages, de dépêches écrites qui sont parfois elles-mêmes le résultat graphique (en français ou en anglais) de messages téléphonés. A l'heure du journal, un texte écrit se déroule devant leurs yeux pour pallier une défaillance de mémoire. Les différents présentateurs d'émissions de variétés ou de jeux ont de plus en plus des notes pour organiser leurs interviews.

Nous retrouvons cette ambiguïté au niveau des produits des enfants ; d'une part, les enfants écrivant par groupes de deux, la gestion de leur écriture se fait au moyen de dialogues oraux à la manière d'un brouillon oral (note 6) ; et d'autre part, leur texte intègre largement des discours directs ou des tournures d'oral strict, en plus des thèmes des produits audiovisuels.

\subsection{Thématique générale des textes}

Si nous nous intéressons à la thématique des textes développés par les six dyades de CM2 (12 enfants), trois de filles, trois de garçons, nous voyons qu'elle est liée à des scénarios de différents produits de l'audiovisuel, jeux vidéo, feuilletons, films fantastiques ou de science-fiction :

- jeux vidéo, fi!ms fantastiques pour Vincent et Andréa,

- !e film Wargames pour Julien et Alexandre, 
- films de guerre du style Guerre des étoiles pour Killy H'ann et Romain,

- un feuilleton comme Super Jamy, ou un film comme Tron pour Enda et Julie,

- des films fantastiques pour Audrey.

Ceci est vrai pour toutes les dyades de garçons et une dyade de filles ; les autres dyades de filles introduisent l'ordinateur dans un contexte assez banal et quotidien de cadeaux de Noël ; si le développement de l'histoire s'apparente au fantastique, il ne semble pas reprendre pour autant un schéma qui serait issu d'un médium audiovisuel.

Bien sûr, nous ne pouvons pas dire que cette thématique soit uniquement audiovisuelle. EUe est sans doute relayée par les revues pour enfants qui suscitent la lecture ou des ouvrages de collections pour jeunes adolescents. Néanmoins aucune référence au livre n'est jamais faite par les enfants au cours de leur rédaction; même l'atmosphère de conte de fées du récit d'une dyade de filles, proche de celle de Cendrillon est plus en prise avec le conte tel qu'il peut être connu par les films de Walt Disney ou autres dessins animés qu'avec le conte véhiculé par l'écrit (note 7).

Prenons comme exemples les débuts de textes de quatre dyades :

a - Dyade Vincent et Andréa : début du deuxième texte, février 1990

le virus

je faisais un jeu de surf sur mon amstrad pc ;les

vagues que je voyais me donnaient soif. j'étais au dernier

tableau là où il fallait passer les vagues de deux mètres,

lorsque soudain, l'ordinateur s'éteignit, c'était un

virus qui avait frappé mon ordinateur.

Le texte est ici en prise directe avec les intérêts des interlocuteurs tels qu'ils peuvent apparaître dans leurs excursus. Nous appelons excursus les moments où les enfants conversent un peu plus librement sans être aux prises directement avec la gestion de la tâche, qu'elle concerne l'utilisation du logiciel graphique ou la création du texte (choix des lexèmes, discussion, correction). Le thème est en congruence avec deux grandes conversations, l'une sur les jeux vidéo (mérites relatifs des différents jeux de monstres, de châteaux hantés et de bolides), l'autre sur les mérites comparés des consoles de jeux ou des ordinateurs. Le thème plus particulier du virus est issu d'une discussion générale au sein de l'atelier et repris par la dyade.

b - Dyade Enda et Julie: deuxième texte, le 20 mars 1990

le tremblement de terre

il était une fois dans le sud de l'Angleterre à Londres,

une jeune fille environ dans les quinze ans. elle était belle. elle avait les yeux bleu, un tout petit nez, des cheveux blons, de belle dents toute blanche et elle mesurait 1M75. elle sape/le cendraa teleéa et elle est dans un 2éme année et elle était sur le point d'être

la plus informer d'informatique de toute l'école.

un jour elle regardit LES INFORMATIONS SUR LE TREMBLEMENT 
DE TERRE QUI ALLAIT BIENTOT SE DEROULER

Le jour du tramblement cendra s'est introduit dans une base

47

militaire ou il y a des million d'ordinateur et elle

regarda autour d'elle...

Ce texte, écrit par une dyade de deux fillettes, nous présente une héroïne qui semble inspirée par différents feuilletons américains ou anglais, mettant en scène soit des adolescents au collège soit une héroïne mi-humaine mi-andrö̈de (Superjamy) à qui sont confiées des missions très délicates du fait de ses pouvoirs paranormaux et de ses facultés exceptionnelles.

Bien que le récit soit très maladroit, nous voyons que l'héroïne correspond à un stéréotype : jeune fille, grande, élancée, blonde, de type anglo-saxon, possédant à la fois une forte culture scientifique et une faculté au delà de l'lntuition, la prémonition ; le thème de l'être humain aux dons surhumains aux prises avec la nature évoque en moins fantastique les aventures de son pendant masculin, Superman.

Le titre même du tremblement de terre est tiré d'une chanson chantonnée par l'une des finllettes à la manière d'un leitmotiv.

Dans un autre texte cette dyade met en scène un ordinateur qui, brusquement, agrippe son interlocutrice humaine et la perd dans ses circuits, scène qui évoque le début du célèbre film Tron.

C - Dyade Julien et Alexandre: premier texte, janvier 1990

1 'ordinateur

c'était un soir, il pleuvait. j'était seul dans ma chambre, je

m'ennuiyais a mourir...

soudain j'eus l'idée de me servir du nouvel ordinateur

que $l$ 'on m'avait offert pour mon anniversaire.

je m'approchais de l'ordinateur pour faire un programme.

je ne me doutais pas encore que ce petit programme, pouvait

être la menace la plus terrible de toute l'humanité!

voici le sens du programme:

mon ordinateur était relié au résau de télecommunication et

je n'en savais rien $1 \ldots . .$.

Le début de l'histoire, ainsi que son développement figurant un conflit mondial évoque le film Wargames ; le héros, qui s'est connecté par mégarde à un réseau de communication militaire, provoque un cataclysme cosmique malgré l'aide d'un savant qui tente de réparer avec lui son erreur. Le récit se termine ensuite très habilement par un retournement de l'espace temps ; projeté sur une planète inconnue, le héros rencontre le fantôme de son ordinateur qui lui permet de construire une machine à remonter te temps. II se retrouve sur la terre au, moment où ses parents lui proposent comme cadeau d'anniversaire un ordinateur et il décide, averti, de le refuser. L'

Jouant sur l'opposition entre le monde et l'anti-monde, le temps et l'antitemps, et pourrait-on dire la matière et $! 1$ anti-matière, le texte exploite des thèmes connus de la science-fiction et même de la science physique 
actuelle. Bien que construit au coup par coup sans planification préalable, le texte est issu d'une mâ̂trise parfaite du genre (littéraire ? filmique ? pouvons-nous vraiment

48

répondre ?). A la manière des séries qui " bouclent " une histoire à chaque épisode, !e retournement s'opère vers la fin et laisse la place possible à un nouveau départ.

d - Dyade Romain et Kilty H'ann : premier texte, Janvier 1990.

1 'ordinateur

il etait une fois un skate board telecommandé, romain kily 'hann on

conçu se superbe skate avec un teile relevé de $52^{\circ}$ concave de $43^{\circ}$ et le nase de $20^{\circ}$.il faisait des holyes flippes $180^{\circ}$ nose pic sur commande. apres 2 ans nous avons conçus une superbe ranpe magnetique avec un canion d'1m 60 d'envirgure avec......

Ce texte, à fonction cryptique (" $i$ comprendront jamais "), particulièrement opaque pour un adulte non initié à la planche à roulettes, est lié aux intérêts des deux garçons qui ont dû faire une véritable traduction pour l'observatrice. En l'écrivant, les garçons évoquent leurs lieux d'entraînement, ainsi qu'une émission sportive télévisée.

R : cette fois-ci, vous êtes sur la Trois à Antenne sport... et voici... vous voyez l'équipe de skate board avec Killy H'ann et Romain, $i$ vont vous parler... no speak (blind(?))... j'avais oublié, mais oui, si vous voulez des renseignements sur Tony Hog, vous allez euh... en Amérique, il est sponsorisé par Powel (chanté)... prochain épisode à 14 heures...

Presque tous les autres récits évoquent des référents filmiques issus de la science-fiction ou du fantastique.

Si nous disons que ces textes sont issus des produits de l'audiovisuel, c'est avant tout en fonction des commentaires, discussions et associations qui environnent le brouillon oral, et également en fonction de la mise en scène du texte lors de sa lecture ou de sa relecture au micro (onomatopées, voix différentes...). Le texte est véritablement oralisé.

\subsection{Chronotope et actants}

Les récits ont en majorité un seul héros humain ; seule une dyade de garçons organise deux textes successifs mettant en scène deux garçons puis deux étudiants. Malgré la dualité des points de vue, un seul héros supporte l'organisation projective de la dyade; l'analyse de l'interaction montre qu'à une ou deux exceptions près, ces héros renvoient à l'organisation imaginaire d'un seul participant, pour des raisons diverses qui ne sont pas toujours clairement décelables (immaturité, absence, affects...). Les groupes de filles, comme leurs camarades masculins développent des récits dont le héros est un garçon ( 8 textes sur 14 ).

Dans les textes des filles, n'interviennent que trois héroïnes : 
- une fillette qui devient femme en épousant un ordinateur puis, après une révolution, un homme de bonne famille dont elle aura sept enfants,

- une adolescente de quinze ans, superwomah, qui grâce à une potion magique calme un volcan en éruption,

-une fillette qui a des cauchemars la nuit.

Les modèles semblent tourner court et les stratégies identitaires des filles (projection / identification) sont complexes puisqu'elles doivent passer par des modèles masculins américanisés ou anglicisés : garçonnet qui reçoit un ordinateur pour Noël, adolescent branché, fou d'ordinateur. Un seul garçon introduit dans son texte le personnage de la sœur du héros qui l'aide dans sa lutte contre les monstres.

Les lieux à partir desquels s'initient les histoires sont des lieux familiers, la maison famliale et le plus souvent la chambre du héros ; l'ordinateur est souvent un outil obtenu pour un anniversaire ou à l'occasion d'une fête. Les enfants partent du quotidien qui se transforme insidieusement en fantastique ; une dyade de filles choisit comme porte d'entrée à l'imaginaire le récit de rêve qui se transforme en cauchemar. L'espace, est un espace plutôt français ou européen, parfois américain ; dans quatre cas, l'histoire se déroule à l'échelon planétaire.

Dans l'organisation des récits, les garçons font lutter leur héros contre différentes catastrophes ou cataclysmes qu'il a lui-même produits ; une des héroïnes féminines tente d'empêcher un tremblement de terre et une éruption volcanique. Les autres dyades luttent contre l'angoisse soulevée par les ordinateurs, angoisses visualisées sous forme de monstres ou non visualisée.

3.3 Confection du texte et du graphisme

Dans la situation d'écriture à laquelle nous faisons référence, les enfants sont aux prises avec plusieurs univers sémiotiques.

Au niveau du produit, le traitement de texte les confronté à l'écrit, dans ses dimensions scripturales et graphiques, au sein d'un genre, le récit, avec conscientisation des phénomènes de mise en page, d'utilisation de polices de caractères, de titrage et de paragraphage. Contrairement à la création du texte et à la cohérence rapprochée, l'orthographe les mobilise peu et relève d'après eux de la compétence du mâ̂tre.

Le logiciel graphique, tout en tes confrontant aussi à des caractéristiques de genre (faire une première page où se mêlent texte, titre, nom d'auteur et dessin, illustrer un texte) les fait revenir à une sémiotique qui a souvent été en partie abandonnée à l'école au cours élémentaire. Néanmoins sur le plan technique, l'utilisation du logiciel graphique est assez complexe ; !es enfants doivent en particulier traiter l'écriture autrement que dans le traitement de texte.

Nous pouvons remarquer que dans cette situation l'imitation joue un grand rôle ; alors qu'ils ne s'interrogent plus sur leurs capacités de création textuelle et qu'ils se placent plutôt dans une optique compétitive (leur texte est meilleur que celui du voisin), les enfants se sentent parfois " nuls" en 
dessin. Ils copient le graphisme d'un camarade qu'ils considèrent comme plus doué, et bien souvent abandonnent la consigne d'illustration personnelle du graphisme par rapport à leur texte au profit de la copie d'un dessin jugé "génial ".

50

Le thème dominant du graphisme est l'éclatement de l'ordinateur ou l'absorption du héros par la machine, thèmes pour images virtuelles d' aujourd' hui.

La sémiotique gestuelle n'a pas été étudiée très précisément car nous n'avons pas utilisé de caméra ; tout au plus évoquerons-nous les conflits pour l'accès au clavier ou à la souris, car ils se manifestent à l'oral. Les enfants restent davantage à leur poste pendant les séances d'écriture ; ils se déplacent plus pendant tes séances de graphisme, bien qu'ils soient alors plus calmes et concentrés.

En CM2, l'écriture longue a nécessité la pérennité des dyades et les groupes sont restés inchangés. Seule l'absence d'un partenaire lors d'une ou plusieurs séances a modifié la situation ; le partenaire présent a tantôt continué le travail commun tantôt modifié le texte initialement créé par la dyade, ce qui a été parfois source de violents conflits. Les élèves se sont réunis par groupes de garçons et groupes de filles. Ceci ne parâtt pas a priori avoir un très grand rapport avec la tâche d'écriture; néanmoins lors des discussions que les enfants ont entre eux dans des moments de détente, ce ne sont pas les mêmes thèmes qui sont abordés : vie familiale, feuilletons de télévision, vedettes, troc, chansons pour les filles ; sport, vie scolaire, informations, reportages, films pour les garçons, ce qui est en relation avec les éléments constitutifs des textes composés.

$\mathrm{Au} C \mathrm{CM} 1$, les enfants se sont également, avec le temps, plutôt regroupés en groupes de filles ou en groupes de garçons, et il nous semble que cela répond au fait que les enfants mobilisent dans la création de leurs textes des bases de données relativement communes, expression d'une expérience (au sens large) partagée, ce qui tend à réduire les discussions en termes d'implicitation et d'explicitation. Nous retrouvons ici l'intrication de différents ordres ; le regroupement des interactants borne déjà l'univers sémantique, l'organisation large du texte. Lorsque nous avons lu les premières lignes de chaque texte à notre groupe de recherche, nos collègues ont reconnu facilement s'ils avaient été écrits par deux garçons ou par deux filles, même si le héros était masculin.

Le dialogue oral constitue l'univers sémiotique au sein duquel se gèrent en partie les différentes actions conjointes : tâche d'écriture, mise en route des programmes, passages de tours de rôle, créations textuelles et corrections éventuelles. Nous y distinguons un niveau premier de stricte planification de l'action en contexte conflictuel ou coopératif, et un niveau second en prise sur le premier, et dévoilant en quelque sorte sur un plan associatif un arrière-fond à partir duquel parlent les enfants.

C'est au sein de ces discours que nous allons maintenant puiser le principal de nos informations concernant tes intérêts et les sources d'inspiration des élèves. Nous pouvons remarquer que ces différentes sémiotiques interfèrent entre elles. Les récits évoquent des jeux vidéo connus, des 
feuilletons de télévision ou des films de cinéma. Le graphisme renvoie majoritairement en miroir au monde de l'ordinateur et les dialogues nous montrent l'intérêt des enfants pour la télévision et la chanson.

51

\section{THÈMES ET VARIATIONS AUTOUR DE L'ORALITÉ}

Les séances d'atelier que nous avons enregistrées, à raison de quatre enregistrements par séance, durant dix séances, duraient en moyenne une heure quinze minutes. Hormis les moments de déconcentration finale et de mise en route toujours délicate du système, une grande partie du temps était donc consacrée au travail d'écriture ou de dessin, dans une tension plus ou moins grande selon !es dyades. Le déroulement des séances de traitement de texte a été souven1 perturbé à la suite d'erreurs faites par les enfants dans le maniement des logiciels ou d'erreurs involontaires de manipulation de l'animatrice. Il existe donc, perceptibles, soit au sein des dyades, soit au sein de l'ate!ier, des blancs, des suspensions de l'activité en cours (charge, écriture, dessin), moments dont certains enfants profitent pour relire leur texte et/ou pour communiquer avec l'observatrice au moyen du magnétophone :

- E: Excusez-nous pour cette interruption ... bonjour mesdames, messieurs, voici notre petit texte (suit une lecture du texte déjà écrit) ... nous reprendrons notre séance la semaine prochaine, les 3/4 heures sont terminés .....(P!us tard dans la séance) notre programme est annulé, nous sommes désolés de ne pouvoir vous lire notre texte ... (Plus tard encore) Au revoir, à la semaine prochaine, adieu, not'programme s'est coupé et nous reprendrons cette séance ... la semaine prochaine.

\subsection{Oral-oralité}

Nous ferons une remarque générale en ce qui concerne l'oralité. Lors du décryptage des enregistrements, il a été parfois difficile malgré le peu d'interlocuteurs en présence, de reconnaître à qui appartenait telle ou telle voix tant !es enfants entrent, dans cette situation, dans une sorte de mimétisme. Nous ne pensons pas que cette imitation soit consciente mais elle est assez fréquente, nous semble-t-il, dans les interactions à deux participants ; l'un d'entre eux ou les deux réciproquement exercent une influence sur l'interlocuteur tant sur le p!an rythmique, qu'intonatif et lexical.

Plus consciente nous parâ̂t être l'utilisation de différents accents par les enfants (surtout les garçons) lorsque leurs créations de texte ; c'est surtout lorsque les enfants répètent le texte finalement choisi par eux pour l'écriture qu'ils le répètent en modifiant leur intonation, leur rythme et égarement souvent leur accent. Parmi les principaux accents que nous avons reconnus, figurent les accents belge, arabe, québécois, anglais. Quelques mots d'anglais sont également parfois utilisés. Les élèves, sans doute un peu lassés d'écrire assez lentement au clavier, se répètent plusieurs fois !e segment à inscrire en modulant de manière ludique leur intonation ; il ne s'agit pas toujours d'un accent catégorisé, mais d'une forme humoristique ou grotesque d'intonation. Parfois les intonations sont personnalisées ; ainsi deux garçons font parler J. Chirac, V.Giscard d'Estaing et le prince d'Angleterre dans une 
séance imaginaire du Bébête show. Les variations de timbre sont également très marquées et affectent surtout les voyelles moyennes (e / $\varepsilon$, $0 / \varnothing$, $\propto /$ et même a / $\alpha$ ), ce qui donne au parler des enfants une phonologie variable qui mériterait sans doute d'être étudiée de

plus près. Il s'agit peut-être d'une capacité à reconnâ̂tre / modifier les formes du français de par la diversité des parlers entendus. Par le biais des ondes et par leur environnement quotidien, les enfants vivent dans une communauté francophone élargie, et les variations de signifiant ont sans doute une fonction d'intercompréhension au sein des sous-communautés. Ces varialions ludiques du signifiant vont parfois jusqu'au jeu de mot.

\subsection{Présentatrices et speakers}

Nous voyons apparâ̂tre dans le dialogue des enfants des scripts assez longs, parodies d'émissions télévisées (programme météo, émission de variétés, émission sportive). Ainsi Enda et Julie jouent-elles une véritable scène d' improvisation.

- E : Bonjour mesdames et messieurs, nous vous parlons en direct de l'ordinateur et avec notre envoyée spéciale, Julia ! voulez-vous nous dire quelques mots ?

- J : (minaudant) Oh non! je suis trop z'émue !

- E : Mais notre public veut que vous nous parliez ! alors, s'il vous plaît, parlez !

- J : (pensant au magnétophone) i faut l'éteindre ! c'est l'histoire d'un tremblement de terre, je voulais vous raconter (puis elle se met à chanter) ... "c'était un tremblement de terre, comme un coup de tonnerre, qui vient nous regarder, nananan nanan, nous déchirer /", c'est l'histoire (elle fait le récit de leur histoire dans laquelle il est justement question d'un tremblement de terre) ... et maintenant je vais vous laisser parler à notre présentatrice. Enda, voulez-vous parler ?

$-\mathrm{E}$ : Eh ben, nous avons de très forts problèmes pour retrouver notre reseau.

- J : Oui, je crois qu'elle a un réseau !

- E : Et quelques pages de publicité 1

- J : (chantonne) Papier toilette, si moelleux et si doux, on n'en fait qu'à sa tête ... quand on a des poux dans les ch'veux... papier toilette qui conduit les ch'veux dans l'paradis ! ... et maintenant, sundy, une barre de chocolat venue vous croquer, vous croquer, dans le ciel ! Sundy, Sundy, ouah, Coca cola c'est ça ! frâ̂cheur d'aujourd'hui, Hollywood chewing gum ! ...

- E : Et nous revenons sur le plateau !

- J : Maintenant si vous téléphonez à quelqu'un, vous devez téléphoner au 46.78.81 et 00 bien sûr ! (suit ensuite une dispute à propos du clavier).

- E : C'est notre nouvelle chanson, "taper sur un bouton... il n'y a qu'à changer le ton, taper sur bouton"... j'ai plus de voix, "casser la voix".

Les fillettes parlent ensuite d'une chanteuse (vraisemblablement Melody) à laquelle Julie a téléphoné. 
Ainsi la fausse chanteuse chante une chanson en relation avec le texte créé par la dyade et la présentatrice rappelle l'exigence du public, avide d'informations. Le baroque de la diversité des publicités est figuré par la coexistence de publicités pour le papier toilette, un produit contre les poux, une barre de chocolat, une boisson et un chewing-gum. Les enfants reproduisent le schéma, interactivité avec le public comprise, des émissions télévisées qu'ils regardent assidûment, émissions qui font alterner de courts segments monologués ou dialogués, des chansons et des publicités. Les chanteurs évoqués sont Melody, Patrick Bruel, Balavoine ... pour ceux dont nous avons pu reconnaître des fragments de textes ou de mélodies. Nous n'avons pas reconnu les fragments en anglais.

Les garçons se passionnent davantage pour les reportages, les émissions sportives ou tes spots météo:

- R. : Je suis en direct sur FR3 ; alors dans le Midi de la France, i f'ra euh ... beau temps, mais des petites averses de ... viendront se glisser dans la fin de l'après-midi et... euh... dans la région du Midi euh... ça s'ra moyen, des p'tites gout'lettes vous tomberont sur la tête et dans les Alpes, dans le Massif Armoricain, beaucoup de pluie et de neige qu'on attendait depuis longtemps ; aujourd'hui, C'est la saint Killy H'ann, on est le 6 février, merci beaucoup, au revoir, à nos studios !

Une fois encore, le script est adapté à la réalité (date du jour et prénom d'un des garçons de la dyade).

\subsection{De la chanson à l'injure}

Pour certains, garçons, mais surtout filles et en dehors de tout schéma long d'émission comme ci-dessus, le dialogue oral débouche sur le chant ou au moins le chantonnement de segments chantés ou de refrains dont les textes vont être repris, déviés et qui serviront de schémas rythmiques à des paroles inventées. Nous en avons un exemple lorsque Julie, qui chantonne la même chanson de Melody depuis le début d'une séance (... "c'est comme un tremblement de terre, comme un coup de tonnerre ... qui vient nous déchirer ... "). Au fur et à mesure que le climat entre les deux partenaires de la dyade se dégrade, nous remarquons des variantes qui peu à peu deviennent scatologiques : "c'est comme la reine d'Angleterre, qui pète dans la mer, à grands coups de tonnerre ". Nous retrouvons ici une des figures de ce que Claude Gaignebet (note 8) appelle le folklore obscène des enfants, le personnage de la Reine d'Angleterre traditionnel dans la chansonnette ou la comptine obscène. Nous avons déjà rencontré dans le dialogue de deux garçons le personnage du prince d'Angleterre!

Ces variantes, et quelques autres de même inspiration, apparaissent dans une séance où une fillette doit participer à l'écriture d'un texte dont elle n'est pas vraiment l'auteur ; elle a été absente durant plusieurs semaines, et sa partenaire a élaboré un texte auquel elle ne semble guère adhérer, mise à part l'introduction du thème du tremblement de terre. Le temps d'une séance, l'enfant va se réfugier dans la parodie, la dérision, le chant avec ses variantes scatologiques et l'injure. Cette gradation dans les conduites verbales est en relation inverse de la 
désaffection du travail en commun sur le texte. L'enfant, qui vit par ailleurs la séparation de ses parents, s'isole dans son monde imaginaire où dominent deux thèmes issus de la chanson, "il n'y a pas que les grands qui rêvent, il n'y a pas que les grands qui ont des sentiments" et "c'est un tremblement de terre, comme un coup de tonnerre ". La scatologie de son parler, ainsi que les injures dont elle abreuve sa partenaire, qui le lui rend bien, nous apparaissent comme une réaction de révolte et de protestation par rapport à sa situation actuelle.

Chez les garçons, l'obscénité apparaît sous forme d'éructations et de halètements divers qui évoquent des fonctions vitales, entrecoupés de courts dialogues à connotation pornographique; ces provocations sont parfois dirigées par l'intermédiaire du micro vers l'observatrice, mais elles servent également, nous semble-t-il, à réguler les tensions à l'intérieur de la dyade. Nous avons retrouvé dans ces groupes d'enfants des attitudes que nous avions déjà rencontrées lors d'une recherche 'préparatoire, de véritables rituels d'injures entre les garçons et également entre les filles (note 9).

\section{EN GUISE DE CONCLUSION}

Nous avons tenté de montrer combien le monde de l'audiovisuel (chansons, images et scénarios de films, scripts d'émissions radiotélévisées ...) influait sur les productions orales (dialogues) et écrites (textes) d'enfants de fin de primaire. Nous avons également insisté sur le fait que des passages, des ponts, existent entre les différents ordres de représentation : un fragment de chanson inspire un titre, un dessin entrevu stimule la continuation du texte, une phrase se transforme en image. Les enfants vivent dans un univers où l'oral, l'image et l'écrit $\mathrm{s}$ 'interpénètrent sans cesse.

Aussi est-il difficile de présenter les relations entre ces ordres en terme d'application simple et directe. Bien qu'ils n'évoquent presque jamais le monde de l'écrit, ces élèves du 7ème arrondissement sont vraisemblablement encore bien des enfants de l'écrit - une certaine aisance dans la continuité des textes en est la preuve - mais ils ne mobilisent pas explicitement cette culture.

Les enfants ( $d u$ moins ces enfants-là) sont loin d'être totalement "sous influence" : ils manifestent à travers la parodie et la moquerie leur distance à l'audiovisuel, ils en détournent les produits et témoignent de la vitalité de la culture orale enfantine d'aujourd'hui.

\section{NOTES}

1

Cf. thèses de 1929 du Cercle de Prague, cf. Change n 3, Seghers / Laffont, Paris, 1970 .

2

Le terrain:

Une école du 7ème arrondissement, rue Montessuy (instituteur J.M. Érédé) et une école du 20éme arrondissement, rue Pelleport (institutrice I. Vajra). Les deux ateliers dépendent de "Paris pour les jeunes ", 110 rue des Amandiers, 75020 Paris, coordinateur informatique J.L. Benguigui. 
En 1989-90, huit mille écoliers parisiens sont allés dans ces ateliers, mais une véritable formation individuelle au traitement de texte et au logiciel graphique n'a été dispensée que dans quelques-uns.

3

Exemples de citations des CM1 (écrit sur leur cahier d'informatique) en réponse à la question "A quoi te fait penser le mot ordinateur ? "

- Sandie : un ordinateur peut effectuer en quelques minutes des calculs qu'un homme mettrait des années à faire, une machine électronique capable d'effectuer des opérations très compliquées. sa vient du dictionnaire HACHETTE. Un ordinateur est comme une petite télévision. Mais au lieu d'avoir une télécommande, il y a un clavier.

- Candice : Un ordinateur me fait penser à une petite télé avec des touches.

- Jean-Michel : Quand on me dit ordinateur sa me fait penser à une machine à écrire plus moderne et sa me fait penser aussi à une calculatrice et à un magnétoscope.

- Jérôme : Le mot ordinateur me fait pensé à une machine très intelligente qui n'oublie nen mes sa me fait penser à un objet de travaille, à mes yeux ses aussi un element de jeu (barré sur la feuille : mes jaimerais beaucoup quond invente un robot télécomandé, à vous de jouer, vive le progré !1!)

4

E. Lage

p. 38 "The primary-school subjects thought of " chifd computer enthusiasts " as - being more outgoing - having more friends - dressing more neatly - coming from a more confortable background.

The primary school subjects consider a girl "computer enthusiast " as - being more cheerful - having more friends - not being a show-off - ooming from a more confortable background.

p. 39 "The overall results indicate that the representations children have of girl's relationship with microcomputers change with age."

p. 39 "For the Junior high students... the girl computer enthusiast is - more of a lonerless interessed in the opposite sex ".

5

Th. Savoyen, corpus recueilli à l'école primaire du Plateau du Moulin de Conflans (78).

Exemples d'évaluations orales d'enfants de CM2 ( 5 spots publicitaires)

- Chrystelle : - "Là j'trouve qu1 z'en parlent trop, i questionnent les gens. Est-ce que vous aimez Kellog's ? Ben oui, j'en prends tous les matins, c'est délicieux au petit déjeuner, j'trouve que c'est un peu bête. "

- Emmanuel : - "Est-ce que c'est la caméra cachée ou pas ? Dans la rue, Monsieur, est-ce que vous aimez Kellog's corn flakes, c'est un peu débile. "

- Emmanuel : "Le Caprice des Dieux, euh... c'est... euh... peut-être que c'est dans un studio et pi euh ... y'a des décors, pi y'a un trucage pour la mer et pi toi quand tu disais d't'à l'heure qu'i y'a une vitrine, c'est exactement pareil que si c'était dans un studio...."

Exemples d'évaluations écrites:

- "Ma pub détestée, Kinder. Pourquo ; parce que quand la dame parle ce n'est pas sincronisé et la publicité est invraisemblable ".

- "Je n'aime pas la pub ou il y a Kinder parce qu'on peut avoir des caries et que la chanson est pas très bien et puis c'est pas bien truqué ". 
Brouillon oral:

mélange de propositions, annulations et progressions successives du texte et de commentaires essentiellement métadiscursifs, métalinguistiques, évaluatifs et argumentatifs (cf. M.-Ch. Pouder 1992b).

Nous nous sommes inspirée pour cette définition des travaux de l'I.T.E.M. (Lebrave - Fuchs - Grésillon) sur les manuscrits d'écrivains et des travaux de Cl. Fabre sur les brouillons d'écoliers, en tentant de cerner les mêmes phénomènes au niveau de l'oral.

7

La seule évocation faite de la lecture dans toutes ces heures d'enregistrement se situe à un moment précis de la création du texte de vincent et Andréa. L'ordinateur du héros s'est arrêté, sans doute atteint d'un virus ; les enfants cherchent une solu-

56

tion pour débloquer la situation Andréa propose que le héros aille dans une librairie et trouve un livre qui lui permettra de comprendre ce qui se passe et de réparer la machine. Vincent refuse ce choix et déclare que le père du héros lui achètera un nouvel ordinateur. Un peu plus tard les deux partenaires s'entendent sur une solution un peu moins coûteuse : le héros se rend dans une agence de maintenance pour faire réparer l'ordinateur. 8

Cl. Gaignebet, Folklore obscène des enfants,

p. 290. Sur plusieurs airs connus dont "La Mère Michel" on trouve, chantés par des enfants de 10-11 ans depuis le début du siècle des textes de ce type :

C'est la reine d'Angleterre

qui s'a fichu par terre

a culotte craqua

et sa lune montra

ou encore

C'est la reine d'Angleterre

qui s'est foutue par terre

en dansant la polka.

$\mathrm{Au}$ bal de l'Opéra

Napoléon ler (variant e: le prince de Galles)

voulut la ramasser,

son pantalon craqua

et la lune se montra.

9

Voici par exemple quelques-uns des mots doux que s'échangent deux fillettes en fin de séance :

- J : Je t'écrabouille le nez !

- E : Tais-toi 1 tu portes la poisse !... et j'te jure, si t'appuies, tu s'ras enfoncée dans la terre ...j'te jure, parfois, on a envie d't'assommer,... J. est une vraie connasse parce que c'est une pute!

- J : E. ... est une vrai connasse parce que c'est une pute

- E : 1 faut qu't'en sortes de ta débilité !... elle est chiante plus que tout !

- J : Pauv'chou!

- E : Perroquet !

- J : ... La ferme! 
- E : J'te tuerai ! ... J. t'es énervante tu sais ,... J. tu énerverais la terre entière ! y'a une claque qui va s'envoler !

- J : Et j'te la donnerai !

\section{BIBLIOGRAPHIE}

M. BAKHTINE, Les genres du discours, in Esthétique de la création verbale, NRF, Gallimard, Paris, 1984 .

J. BRUNER, Le développement de l'enfant : Savoir faire, savoir dire, Paris: Presses Universitaires de France, 1983.

L.J. CALVET, Langue, corps, société, Payot, Paris, 1979.

Change $\mathrm{n}^{\circ} 3$, Le Cercle de Prague, Pour une science théorique de l'écriture, éditions Seghers / Laffont, Paris 1970.

F. FRANÇOIS, Morphologie, syntaxe et discours, in Problèmes de Psycholinguistique, Textes rassemblés par R. Rondal et J.-P. Thibaut, collection Psychologie et Sciences Humaines, Mardaga éditeurs, Bruxelles, 1987.

57

Cl. GAIGNEBET, Le folklore obscène des enfants, Maisonneuve et Larose, Paris, 1974 .

R. JONES, Le petit enfant face au livre, analyse des gestes de repérage, Bull. d'Audiophonologie, Ann. Sc. Univ. Franche-Comté, vol. IV, NS, n 4-5, 1988.

E. LAGE, Boys, girls and Microcomputing, European Journal of Psychology of Education, vol. VI, $\mathrm{n}^{\circ} 1,1991$.

Th. MEYER, Usages et représentations du traitement de texte chez les enseignants-chercheurs et chercheurs, in Texte et ordinateur : les mutations du lire-écrire, sous la direction de J. Anis et J.L. Lebrave, Actes du colloque interdisciplinaire tenu à l'Université Paris X - Nanterre, 6-7-8 Juin 1990, vol. hors série de la revue Linx, publication du Centre de Recherches Linguistiques de Paris X - Nanterre, éditions de l'Espace Européen, La Garenne-Colombes, France, 1991.

M.-Ch. POUDER, N. MARTY-TEMPORAL, J. ZWOBADA-ROSEL, Propos d'ordinateur, IVe colloque DFLM, Genève, Diversifier l'enseignement du français écrit, sous la direction de B.Schneuwly, Delachaux et Niestlé, Neuchâtel Paris, 1992 (a).

M.-Ch. POUDER, Produire une récit à deux sur ordinateur ; entre programmation de l'écriture et accident du dialogue, CAlap $n^{\circ} 9$, Réécriture et interactivité en milieu scolaire, URA CNRS 1031 - Université René Descartes, $1992(\mathrm{~b})$.

M.-Ch. POUDER, Écrire avec l'ordinateur au CM2, temps et cullure à partager, Vème colloque DFLM, Montréal, L'hétérogénéité des apprenants : un défi pour la classe de français, sous la direction de M. Lebrun et M. Ch. Paret, Delachaux et Niestlé, Neuchâtel-Paris, 1993.

Pour la science $\mathrm{n}^{\circ}$ spécial, Le Cerveau et la Pensée, n¹81, nov. 1992,

p. 80 : A. Damasio et H. Damasio, Le cerveau et le langage,

p. 28: G. Fischbach, Le cerveau et la pensée.

J. RABAIN-JAMIN, E. SABEAU-JOUANNET, Playing with pronouns in French maternal speech to prelingual infants, J. Child Language 16, 1989.

J. RABAIN-JAMIN, Conduites de soutien et gestion du dialogue entre mères et enfants africains et français à Paris, article présenté lors d'une intervention au ler colloque international Communication, Sciences du Langage et Sciences 
Humaines, L'analyse des interactions, Université de Provence, Aix-en-Provence, sept. 1991.

Th. SAVOYEN, La publicité chez l'enfant, évaluation de cinq spots publicitaires à l'écrit puis à l'oral dans trois classes (maternelle, CE1, CM2), Mémoire pour le Certificat de capacité d'orthophonie, année 88-89, Université de Paris VI Salpétrière. 